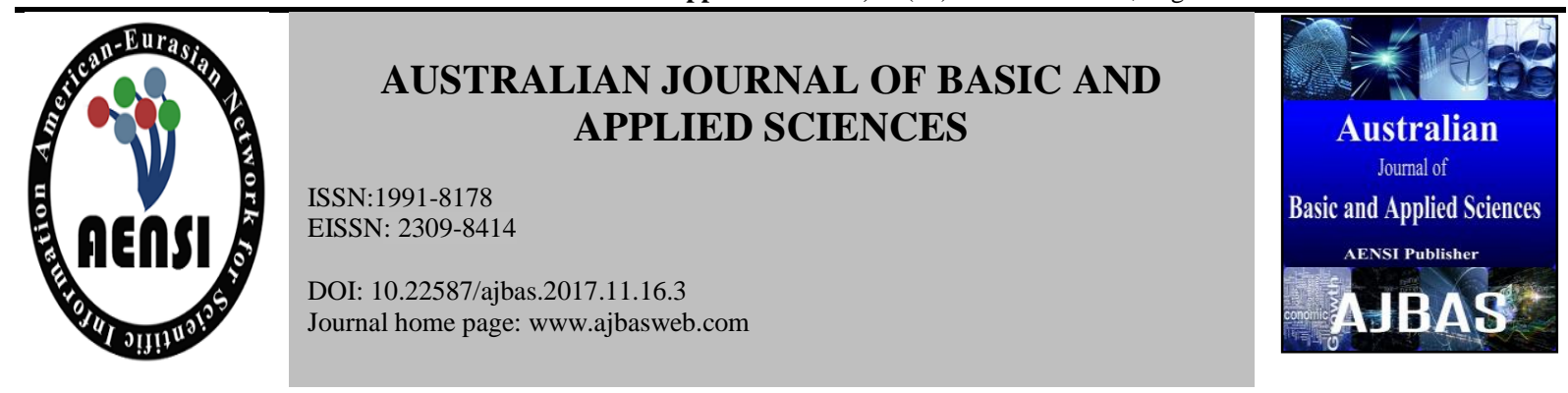

\title{
Proposed Algorithm for Gumbel Distribution Estimation
}

Omar Abdulmohsin Ali

Assistant Professor, Department of Statistics, College of Administration and Economic, University of Baghdad, Baghdad City, Iraq.

Address For Correspondence:

Omar Abdulmohsin Ali, Assistant Professor, Department of Statistics, College of Administration and Economic, University of Baghdad, Baghdad City, Iraq.

E-mail: dromar72@coadec.uobaghdad.edu.iq

\section{A R T I CLE INFO}

Article history:

Received 12 October 2017

Accepted 22 December 2017

Available online 15 January 2018

Keywords:

Gumbel Distribution, Maximum

Likelihood, Jackknife, Artificial

intelligence, Hybrid methods

Simulation.

\begin{abstract}
A B S T R A C T
Gumbel distribution was dealt with great care by researchers and statisticians. There are traditional methods to estimate two parameters of Gumbel distribution known as Maximum Likelihood, the Method of Moments and recently the method of re-sampling called (Jackknife). However, these methods suffer from some mathematical difficulties in solving them analytically. Accordingly, there are other non-traditional methods, like the principle of the nearest neighbors, used in computer science especially, artificial intelligence algorithms, including the genetic algorithm, the artificial neural network algorithm, and others that may to be classified as meta-heuristic methods. Moreover, this principle of nearest neighbors has useful statistical features. The objective of this paper is thus to propose a new algorithm where it allows getting the estimation of the parameters of Gumbel probability distribution directly. Furthermore, it overcomes the mathematical difficulties in this matter without need to the derivative of the likelihood function. Taking simulation approach under consideration as empirical experiments where a hybrid method performs optimization of these three traditional methods. In this regard, comparisons have been done between the new proposed method and each pair of the traditional methods mentioned above by efficiency criterion Root of Mean Squared Error (RMSE). As a result, (36) experiments of different combinations of initial values of two parameters $(\lambda$ : shift parameter and $\theta$ : scale parameter) in three values that take four different sample sizes for each experiment. To conclude, the proposed algorithm showed its superiority in all simulation combinations associated with all sample sizes for the two parameters $(\lambda$ and $\theta)$. In addition, the method of Moments was the best in estimating the shift parameter $(\lambda)$ and the method of Maximum Likelihood was in estimating the scale parameter $(\theta)$.
\end{abstract}

\section{INTRODUCTION}

Much attention has been given to the application of Gumbel distribution because of its importance as known "the extreme value threshold" which utilized in global warming, rain falls and dust storms. Gumbel, E.J., (1954) was the first who dealt with this distribution behavior. The class of continuous distributions Extreme value has two types of Extreme value distributions according to (maxima or minima) that dealt with these situations. Gumbel (Type I) distribution is the maximum value that referred to in this paper. The motivation of this paper is that limited researches that dealt with the derivative difficulties associated with this type of distribution in using Maximum Likelihood. Moreover, the slowly resampling procedure in Jackknife Method indicated as disadvantage. The need to estimate both parameters of Gumbel distribution in a modern fast method based on seeking an optimal estimation as a target point. This approach done by reduction the distance between the traditional estimates from the target value which make the achievement to the required estimate faster and slightly.

\footnotetext{
Open Access Journal

Published BY AENSI Publication
}

(C) 2017 AENSI Publisher All rights reserved

This work is licensed under the Creative Commons Attribution International License (CC BY).

http://creativecommons.org/licenses/by/4.0/

\section{Open Access}

To Cite This Article: Omar Abdulmohsin Ali., Proposed Algorithm for Gumbel Distribution Estimation. Aust. J. Basic \& Appl. Sci., 11(16): 17-24, 2017 


\section{MATERIALS AND METHODS}

\subsection{Gumbel Distribution:}

Al-Adilee and Mohamed (2014) argued Gumbel distribution as one of the Extreme Value distributions such as type II - Frechet distribution and type III - Weibull distribution.

The random variable y said to have Gumbel distribution with two parameters $\lambda$ which represents the shift parameter and $\theta$ that represents the scale parameter. If it has the following right skewed probability density function as presented by Aydin and Şenoğlu (2015):

$f(y ; \lambda, \theta)=\frac{e^{-\left(\frac{y-\lambda}{\theta}\right)}}{\theta} \exp \left[-e^{-\left(\frac{y-\lambda}{\theta}\right)}\right] \quad ;$ where is: $\quad y, \lambda \in R \quad, \quad \theta>0$

Aydinand Şenoğlu (2015) obtained the Cumulative Probability Function as:

$F(y)=\operatorname{Pr}(Y \leq y)=\exp \left[-e^{-\left(\frac{y-\lambda}{\theta}\right)}\right]$

\subsection{Maximum Likelihood Method (ML):}

The method of maximum likelihood has been acknowledged by Raynal-Villasenor (2012) as one of the best methods for parameter estimation of probability distribution functions. The properties of its estimators like the invariance property. The maximum likelihood function can be given as described by Mahdi et al (2005).

$L(y ; \lambda, \theta)=\left(\frac{1}{\theta}\right)^{n} e^{-\sum_{i=1}^{n}\left(\frac{y i-\lambda}{\theta}\right)} \exp \left[-\sum_{i=1}^{n} e^{-\left(\frac{y i-\lambda}{\theta}\right)}\right]$

But, after taking logarithm:

$\ln L(y ; \lambda, \theta)=-n \ln (\theta)-\sum_{i=1}^{n}\left(\frac{y_{i}-\lambda}{\theta}\right)-\sum_{i=1}^{n} \exp \left(\frac{y_{i}-\lambda}{\theta}\right)$

with partial derivatives below.

$$
\frac{\partial \ln L}{\partial \lambda}=\lambda+\theta \ln \left[\frac{\sum_{i=1}^{n} \exp \left(-\frac{y_{i}}{\theta}\right)}{n}\right]
$$

$\frac{\partial \ln L}{\partial \lambda}=0$

$$
\rightarrow \hat{\lambda}_{M L}=-\theta \ln \left[\frac{\sum_{i=1}^{n} \exp \left(-\frac{y_{i}}{\theta}\right)}{n}\right]
$$

Or: $\quad \hat{\lambda}_{M L}=-\frac{\theta}{n} \ln \left[\sum_{i=1}^{n} \exp \left(-\frac{y_{i}}{\theta}\right)\right]$

$$
\frac{\partial \ln L}{\partial \theta}=\sum_{i=1}^{n} y_{i} \exp \left(\frac{y_{i}}{\theta}\right)-(\bar{y}+\lambda) \exp \left(\frac{\lambda}{\theta}\right)
$$

$\frac{\partial \ln L}{\partial \theta}=0$ 
$\rightarrow \hat{\theta}_{M L}=\bar{y}-\frac{\sum_{i=1}^{n} y_{i} \exp \left(\frac{y_{i}}{\hat{\theta}}\right)}{\sum_{i=1}^{n} \exp \left(\frac{y_{i}}{\hat{\theta}}\right)}$

Since equations (5) \& (7) are very difficult to be solved, hence, iterative numerical Newton-Raphson technique is required in this case to get $\left(\hat{\lambda}_{M L}, \hat{\theta}_{M L}\right)$ respectively.

\subsection{Method of Moments (MOM):}

The population moment of order $\mathbf{r}$ can be obtained by Mahdi and Cenac (2005):

$\mu_{r}=E\left(y^{r}\right)$

While the sample moment of order $\mathbf{r}$ is:

$m_{r}=\frac{1}{n} \sum_{i=1}^{n}\left(y_{i}^{r}\right)$

Therefore, the first moment will be:

$\mu_{1}=E(y)=\lambda+\gamma \theta$

Where is : $\gamma=0.5772$ Euler-Mascheroni constant as referred to by Clarke (2002) \& Yousef and Sameer (2014).

$$
\frac{\sum_{i=1}^{n} y_{i}}{n}=E(y) \quad \rightarrow \quad \bar{y}=\lambda+\gamma \theta
$$

$\therefore \lambda=\bar{y}-\gamma \theta$

Moreover, the second moment will be:

$$
\operatorname{Var}(y)=E\left(y^{2}\right)-[E(y)]^{2}=\frac{\pi^{2} \theta^{2}}{6}
$$

Where is : $\gamma=0.5772$ Euler's constant.

$$
\begin{aligned}
& \frac{\sum_{i=1}^{n} y_{i}^{2}}{n}=E\left(y^{2}\right) \quad \rightarrow \quad \frac{\sum_{i=1}^{n} y_{i}^{2}}{n}=\operatorname{Var}(y)+[E(y)]^{2} \\
& \therefore \frac{\sum_{i=1}^{n} y_{i}^{2}}{n}=\frac{\pi^{2} \theta^{2}}{6}+[\lambda+\gamma \theta]^{2}
\end{aligned}
$$

Where is : $\pi=\frac{22}{7}$

By solving the last equation above we get the moment estimation of $(\theta)$ is:

$\hat{\theta}_{M O M}=\frac{\sqrt{6}}{\pi} S$

S: the standard deviation of the data. The moment estimation of $(\lambda)$ is: 


$$
\begin{gathered}
\hat{\lambda}=\bar{y}-\gamma \frac{\sqrt{6}}{\pi} S \\
\rightarrow \hat{\lambda}_{M O M}=\bar{y}-0.449 S
\end{gathered}
$$

\subsection{Jackknife Method (Jack.):}

This method is based on successive elimination and repetitions of the values of the random variable in order to get the partial samples that obtained from that elimination. This method is described by Eforn (1979) as "bootstrap method". Hall et al (2004) assumed that there is random sample of size (n) of the observations follows random variable which distributed as Gumbel distribution, then many partial samples can be formed, each of them consists of a number of observations so that:

$y_{i j} ; \mathrm{i}=1,2,3, \ldots, \mathrm{n}-\mathrm{k} \quad ; \quad \mathrm{j}=1,2,3, \ldots, \mathrm{m}$

Where is $(\mathrm{k})$ : represents the number of the eliminating observations at each time.

$(\mathrm{n}-\mathrm{k})$ : the partial sample size resulting after elimination.

$(\mathrm{m})$ : the number of partial samples occurred.

$$
\hat{\varphi}_{\text {Jack }}=n \hat{\varphi}_{0}-(n-1) \sum_{i=1}^{m} \frac{\hat{\varphi}_{(i) 0}}{m}
$$

Where is: $\hat{\varphi}_{0}$ is the initial value got from some estimation method.

$\hat{\varphi}_{(i) 0}$ : the initial value got from some estimation method at $\left(\mathrm{i}^{\text {th }}\right)$ iteration.

\subsection{The Proposed Method (Prop.):}

Iterative meta-heuristic algorithms are the most rapid widespread modern approaches with computer aided in the world.

The core idea is how to find wide verities of point estimations at once which need only to minimize (or maximize) one objective function (say minimize RMSE) rather than derivatives. The second important thing is how to find a sub-set of the possible estimates for the parameters applied to all possible combinations resulting from the initial estimations. After that all the estimates are compared on the basis of the comparison criterion to determine the optimal combination of estimates and then re-combination approach repeated for a number of times until getting the suitable alternatives which determine the required estimate.

Step 1: Preparing initial values for the both pair parameters $(\lambda, \theta)$ form each of the traditional methods previously obtained, i.e., ML, MOM and Jackknife as "inputs".

Step 2: Distinguish the best estimation method among those traditional estimates (say; $\lambda_{0}, \theta_{0}$ ) based on (RMSE) criterion.

Step 3: Conduct a region that formed by huge number of points (say; $\lambda_{0 \mathrm{i}}, \theta_{0 \mathrm{i}}$ ) $; \mathrm{i}=1,2,3, \ldots, 100$ that falls into the intervals:

$$
\left\{\lambda_{0 \mathrm{i}} \in\left(\lambda_{0 \mathrm{i}} \pm 0.5\right), \theta_{0 \mathrm{j}} \in\left(\theta_{0 \mathrm{j}} \pm 0.5\right)\right\}
$$

with all their possible combinations that could be formed.

Step 4: Compare the selected best method $\left(\lambda_{0}, \theta_{\mathrm{o}}\right)$ got in step(2) against the $\left(\lambda_{0 \mathrm{i}}, \theta_{0 \mathrm{j}}\right)$ got in step(3) to obtain the optimal combination among them based on (RMSE) criterion.

Step 5:Continuing subsequent of candidate points iteratively until getting the final estimations $\left(\hat{\lambda}_{\text {prop. }}, \hat{\theta}_{\text {prop }}\right)$ by using the stopping rule in terms of Total Squared Error (TSE) as below.

$T S E=\frac{\left(\hat{\lambda}_{i}-\hat{\lambda}_{i-1}\right)^{2}+\left(\hat{\theta}_{i}-\hat{\theta}_{i-1}\right)^{2}}{2}$

The optimality will be got at convergence when stopping iff: TSE $\leq 0.0001$ 


\section{Comparison Criterion:}

Suppose $(\varphi)$ be one of the two parameters of Gumbel distribution $(\lambda)$ or $(\theta)$. Let (rep) be the replications.

Root of Mean Squared Error (RMSE):

$R M S E=\sqrt{\frac{\sum_{i=1}^{r e p}\left\{\varphi_{i}-\hat{\varphi}_{i}\right\}^{2}}{\text { rep }}}$

\section{Simulation:}

Empirical experiments performed by (36) simulations assembled by conducting the combination of parameters as follows:
$\lambda: 2 \quad 3 \quad 5$
$\begin{array}{llll}\boldsymbol{\theta}: & 0.5 & 1.0 & 2.0\end{array}$
n: $\quad \begin{array}{llll}15 & 30 & 60 & 150\end{array}$

Consequently, (y) can be generated as a random variable followed Gumbel distribution with "Inverse Cumulative Distribution Function" by the following steps described by Mohammed (2011):

$$
\begin{aligned}
& F(y)=\exp \left[-e^{-\left(\frac{y-\lambda}{\theta}\right)}\right] \\
& \ln [F(y)]=-\exp \left[-\left(\frac{y-\lambda}{\theta}\right)\right] \\
& \ln \{-\ln [F(y)]\}=-\left(\frac{y-\lambda}{\theta}\right) \\
& -\ln \{-\ln [F(y)]\}=\frac{y-\lambda}{\theta}
\end{aligned}
$$

Let: $\mathrm{U}=\mathrm{F}(\mathrm{t})$

where is $\mathrm{U}$ : random variable belongs to $[0,1]$.

Hence,

$$
y=\lambda+\theta\{-\ln [-\ln (U)]\}
$$

The numerical results in the following tables: (1, 2, 3 and 4) as below.

\section{Conclusions:}

Four estimation methods were compared. The comparison was performed with respect to their (RMSE) criterion. From the previous numerical results obtained from simulation experiments, the following conclusions achieved:

i.) New areas were explored in the estimation space and the proposed algorithm showed its superiority. It was obvious that proposed method is the best method for both parameters based on (RMSE) criterion and all sample sizes.

ii.) (MOM) method got better results gradually as sample size grew for $\operatorname{RMSE}(\lambda)$ from Table (3) compared with (ML) \& (Jack). However, it got worse results gradually as sample size grew for RMSE( $\theta$ ) from Table (4) compared with (ML) \&(Jack).

iii.) It can also be noticed from the experimentation results of RMSE for shift parameter ( $\lambda$ ) that (MOM) is better than its traditional competitor (ML) \& (Jack.) for all sample sizes. Alternatively, the RMSE of scale parameter $(\theta)$ results indicated that (ML) is better than $(\mathrm{MOM}) \&(\mathrm{Jack}$.) for all sample sizes.

iv.) The worst combination of the two estimated parameters was $(\lambda=3, \theta=2)$ for all sample sizes according to Table (3) and Table (4) regardless to the estimation method.

v.) The best results according to Table (4) for all sample sizes was $(\theta=0.5)$ regardless to $(\lambda)$ value and estimation method. 
Table 1: Estimated $(\lambda)$

\begin{tabular}{|c|c|c|c|c|c|c|}
\hline \multirow[t]{2}{*}{$\mathrm{n}$} & \multirow[t]{2}{*}{$\bar{\lambda}$} & \multirow[t]{2}{*}{$\theta$} & \multicolumn{4}{|c|}{ Estimation Method } \\
\hline & & & ML & MOM & Jack & Prop. \\
\hline 15 & 2 & 0.5 & 2.01687295 & 2.00013710 & 1.98485259 & 2.00000594 \\
\hline 15 & & 1.0 & 2.03526052 & 2.00795115 & 1.94935496 & 2.00381237 \\
\hline 15 & & 2.0 & 2.12073258 & 2.06315467 & 1.94771107 & 2.01882522 \\
\hline 15 & 3 & 0.5 & 3.02418095 & 3.00995118 & 2.99213706 & 3.00008071 \\
\hline 15 & & 1.0 & 3.05102813 & 3.03381198 & 2.99507406 & 3.00457684 \\
\hline 15 & & 2.0 & 3.16110025 & 3.08266404 & 2.99374980 & 3.03444466 \\
\hline 15 & 5 & 0.5 & 5.02386600 & 5.00841508 & 5.00158420 & 5.00009944 \\
\hline 15 & & 1.0 & 5.03931304 & 5.00635059 & 4.96573863 & 5.00383792 \\
\hline 15 & & 2.0 & 5.12493827 & 5.05021937 & 5.03710306 & 5.01925103 \\
\hline 30 & 2 & 0.5 & 2.01163546 & 2.00631791 & 1.99867497 & 1.99998371 \\
\hline 30 & & 1.0 & 2.02226752 & 2.00755722 & 1.99785651 & 2.00044221 \\
\hline 30 & & 2.0 & 2.03146848 & 2.00917962 & 1.98271196 & 2.00394460 \\
\hline 30 & 3 & 0.5 & 3.01278058 & 3.00735583 & 3.00124696 & 3.00004512 \\
\hline 30 & & 1.0 & 3.00747531 & 2.99996731 & 2.98399509 & 3.00041554 \\
\hline 30 & & 2.0 & 3.05300313 & 3.03349474 & 3.00033677 & 3.00864270 \\
\hline 30 & 5 & 0.5 & 5.00952609 & 5.00447302 & 4.99726854 & 4.99993293 \\
\hline 30 & & 1.0 & 5.03281973 & 5.01933509 & 5.00792990 & 5.00079095 \\
\hline 30 & & 2.0 & 5.03361676 & 5.01845498 & 4.98804012 & 5.00087041 \\
\hline 60 & 2 & 0.5 & 2.00504952 & 2.00202366 & 1.99961839 & 1.99997438 \\
\hline 60 & & 1.0 & 2.02249332 & 2.01733530 & 2.01132036 & 2.00016600 \\
\hline 60 & & 2.0 & 2.04498668 & 2.03467061 & 2.02264168 & 2.00252599 \\
\hline 60 & 3 & 0.5 & 3.01124667 & 3.00866765 & 3.00566064 & 3.00007537 \\
\hline 60 & & 1.0 & 3.02249333 & 3.01733530 & 3.01132108 & 3.00016662 \\
\hline 60 & & 2.0 & 3.04498666 & 3.03467061 & 3.02264021 & 3.00252503 \\
\hline 60 & 5 & 0.5 & 5.01124668 & 5.00866765 & 5.00566125 & 5.00007737 \\
\hline 60 & & 1.0 & 5.02249333 & 5.017333530 & 5.01132064 & 5.00016694 \\
\hline 60 & & 2.0 & 5.04498671 & 5.03467061 & 5.02264388 & 5.00252673 \\
\hline 150 & 2 & 0.5 & 2.00205414 & 2.00224838 & 1.99982111 & 1.99995004 \\
\hline 150 & & 1.0 & 2.00793936 & 2.00560769 & 2.00338195 & 1.99994432 \\
\hline 150 & & 2.0 & 2.00706312 & 2.00341928 & 1.99838890 & 2.00024806 \\
\hline 150 & 3 & 0.5 & 3.00176578 & 3.00085482 & 2.99959749 & 3.00006455 \\
\hline 150 & & 1.0 & 3.00178400 & 3.00193642 & 2.99709763 & 2.99986241 \\
\hline 150 & & 2.0 & 3.01024694 & 3.01165291 & 3.00113711 & 3.00013413 \\
\hline 150 & 5 & 0.5 & 5.00277807 & 5.00231985 & 5.00061546 & 4.99989187 \\
\hline 150 & & 1.0 & 5.00619542 & 5.00559623 & 5.00161851 & 4.99980984 \\
\hline 150 & & 2.0 & 5.01528287 & 5.01375377 & 5.00694093 & 4.99997528 \\
\hline
\end{tabular}

Table 2: Estimated $(\theta)$

\begin{tabular}{|l|l|l|l|l|l|l|}
\hline $\mathrm{n}$ & $\lambda$ & $\theta$ & \multicolumn{5}{|l|}{ Estimation Method } \\
\cline { 4 - 8 } & & & ML & MOM & Jack & Prop. \\
\hline 15 & 2 & 0.5 & 0.47340057 & 0.48716625 & 0.50070369 & 0.49985106 \\
\hline 15 & & 1.0 & 0.94519179 & 0.98825839 & 0.98193606 & 1.00017895 \\
\hline 15 & & 2.0 & 1.84999516 & 1.90876550 & 1.96594206 & 1.99643857 \\
\hline 15 & 3 & 0.5 & 0.46912211 & 0.48861856 & 0.49072002 & 0.49990848 \\
\hline 15 & & 1.0 & 0.92152371 & 0.98803207 & 0.99653682 & 1.00002829 \\
\hline 15 & & 2.0 & 1.91431798 & 1.92881302 & 2.01233153 & 1.99039917 \\
\hline 15 & 5 & 0.5 & 0.46899180 & 0.48389307 & 0.49623979 & 0.49992426 \\
\hline 15 & & 1.0 & 0.93386602 & 0.97064593 & 0.98903082 & 1.00022880 \\
\hline 15 & & 2.0 & 1.89079434 & 1.94936096 & 2.00435544 & 1.99033960 \\
\hline 30 & 2 & 0.5 & 0.48035508 & 0.48764823 & 0.49583896 & 0.50008038 \\
\hline 30 & & 1.0 & 0.96627305 & 0.96912808 & 0.99741166 & 1.00030171 \\
\hline 30 & & 2.0 & 1.94983898 & 1.98292174 & 2.01733912 & 2.00144285 \\
\hline 30 & 3 & 0.5 & 0.47903445 & 0.48885121 & 0.49501202 & 0.50001098 \\
\hline 30 & & 1.0 & 0.96596632 & 0.99492486 & 0.99893773 & 0.99984379 \\
\hline 30 & & 2.0 & 1.91652650 & 1.96886023 & 1.97762203 & 1.99896717 \\
\hline 30 & 5 & 0.5 & 0.48757920 & 0.50025411 & 0.50385717 & 0.50009175 \\
\hline 30 & & 1.0 & 0.97170574 & 0.97919716 & 1.00504403 & 1.00003086 \\
\hline 30 & & 2.0 & 1.90885744 & 1.95988280 & 1.96999883 & 1.99997218 \\
\hline 60 & 2 & 0.5 & 0.49248828 & 0.49609373 & 0.50146617 & 0.49995777 \\
\hline 60 & & 1.0 & 0.99842427 & 1.00069664 & 1.01524060 & 0.99999819 \\
\hline 60 & & 2.0 & 1.99684850 & 2.00139328 & 2.03047637 & 2.00013115 \\
\hline 60 & 3 & 0.5 & 0.49921213 & 0.50034832 & 0.50761971 & 0.49979508 \\
\hline 60 & & 1.0 & 0.99842425 & 1.00069664 & 1.01524234 & 1.00002281 \\
\hline 60 & & 2.0 & 1.99684849 & 2.00139328 & 2.03047585 & 2.00013054 \\
\hline 60 & 5 & 0.5 & 0.49921213 & 0.50034832 & 0.50761975 & 0.49979512 \\
\hline 60 & & 1.0 & 0.99842425 & 1.00069664 & 1.01523921 & 1.00002137 \\
\hline
\end{tabular}


Omar Abdulmohsin Ali, 2017

Australian Journal of Basic and Applied Sciences, 11(16) December 2017, Pages: 17-24

\begin{tabular}{|l|l|l|l|l|l|l|}
\hline 60 & & 2.0 & 1.99684853 & 2.00139328 & 2.03047793 & 2.00013090 \\
\hline 150 & 2 & 0.5 & 0.49752254 & 0.49835525 & 0.50096611 & 0.49996120 \\
\hline 150 & & 1.0 & 0.99488753 & 0.99351863 & 1.00167836 & 1.00010323 \\
\hline 150 & & 2.0 & 1.98449844 & 1.98401702 & 1.99830001 & 1.99986705 \\
\hline 150 & 3 & 0.5 & 0.49612463 & 0.49600425 & 0.49957927 & 0.49999594 \\
\hline 150 & & 1.0 & 0.99265964 & 0.99957968 & 0.99938075 & 1.00022550 \\
\hline 150 & & 2.0 & 1.98880900 & 2.00433168 & 2.00225519 & 1.99998444 \\
\hline 150 & 5 & 0.5 & 0.49574681 & 0.49821113 & 0.49933749 & 0.50008122 \\
\hline 150 & & 1.0 & 0.99518262 & 1.00318525 & 1.00212865 & 0.99994279 \\
\hline 150 & & 2.0 & 1.99018287 & 1.99748582 & 2.00389676 & 2.00012282 \\
\hline
\end{tabular}

Table 3: $\operatorname{RMSE}(\lambda)$

\begin{tabular}{|c|c|c|c|c|c|c|}
\hline \multirow[b]{2}{*}{$\mathrm{n}$} & \multirow[b]{2}{*}{$\lambda$} & \multirow[b]{2}{*}{$\theta$} & \multicolumn{4}{|c|}{ Estimation Method } \\
\hline & & & ML & MOM & Jack & Prop. \\
\hline 15 & 2 & 0.5 & 0.12255397 & 0.10585322 & 0.14457355 & 0.00125518 \\
\hline 15 & & 1.0 & 0.24795257 & 0.22094560 & 0.27513064 & 0.00889251 \\
\hline 15 & & 2.0 & 0.51889817 & 0.46036395 & 0.57418386 & 0.09268990 \\
\hline 15 & 3 & 0.5 & 0.11831718 & 0.10386042 & 0.13873913 & 0.00120366 \\
\hline 15 & & 1.0 & 0.25002239 & 0.22397198 & 0.26923814 & 0.00861155 \\
\hline 15 & & 2.0 & 0.53366501 & 0.47365601 & 0.57070566 & 0.09854717 \\
\hline 15 & 5 & 0.5 & 0.13440976 & 0.11656101 & 0.14389626 & 0.00138880 \\
\hline 15 & & 1.0 & 0.25414928 & 0.22284453 & 0.27887802 & 0.00966722 \\
\hline 15 & & 2.0 & 0.50376716 & 0.43452776 & 0.57415456 & 0.07982590 \\
\hline 30 & 2 & 0.5 & 0.07945469 & 0.07070697 & 0.08017592 & 0.00126835 \\
\hline 30 & & 1.0 & 0.16580789 & 0.15441065 & 0.16548858 & 0.00203270 \\
\hline 30 & & 2.0 & 0.32379334 & 0.29653698 & 0.33281060 & 0.02256790 \\
\hline 30 & 3 & 0.5 & 0.08463460 & 0.07882035 & 0.08423825 & 0.00128835 \\
\hline 30 & & 1.0 & 0.16925912 & 0.16004951 & 0.16921010 & 0.00160620 \\
\hline 30 & & 2.0 & 0.31546496 & 0.29472594 & 0.31499696 & 0.02736278 \\
\hline 30 & 5 & 0.5 & 0.08600126 & 0.07994527 & 0.08507069 & 0.00124837 \\
\hline 30 & & 1.0 & 0.17399038 & 0.16013726 & 0.17130431 & 0.00227011 \\
\hline 30 & & 2.0 & 0.33111669 & 0.30875702 & 0.32607332 & 0.03169727 \\
\hline 60 & 2 & 0.5 & 0.05968998 & 0.05627056 & 0.05939350 & 0.00125122 \\
\hline 60 & & 1.0 & 0.11492629 & 0.10795750 & 0.11408868 & 0.00128772 \\
\hline 60 & & 2.0 & 0.22985251 & 0.21591501 & 0.22817479 & 0.00702708 \\
\hline 60 & 3 & 0.5 & 0.05746313 & 0.05397875 & 0.05704409 & 0.00112729 \\
\hline 60 & & 1.0 & 0.11492626 & 0.10795750 & 0.11408708 & 0.00128733 \\
\hline 60 & & 2.0 & 0.22985254 & 0.21591501 & 0.22817593 & 0.00702771 \\
\hline 60 & 5 & 0.5 & 0.05746314 & 0.05397875 & 0.05704514 & 0.00112762 \\
\hline 60 & & 1.0 & 0.11492628 & 0.10795750 & 0.11408791 & 0.00128801 \\
\hline 60 & & 2.0 & 0.22985259 & 0.21591501 & 0.22817880 & 0.00702704 \\
\hline 150 & 2 & 0.5 & 0.03815429 & 0.03506439 & 0.03793725 & 0.00136809 \\
\hline 150 & & 1.0 & 0.07296097 & 0.06973584 & 0.07283006 & 0.00119310 \\
\hline 150 & & 2.0 & 0.14338600 & 0.13869797 & 0.14408988 & 0.00141310 \\
\hline 150 & 3 & 0.5 & 0.03584650 & 0.03467449 & 0.03602393 & 0.00125502 \\
\hline 150 & & 1.0 & 0.07130207 & 0.07001158 & 0.07194569 & 0.00123789 \\
\hline 150 & & 2.0 & 0.15808690 & 0.15117713 & 0.15705439 & 0.00142015 \\
\hline 150 & 5 & 0.5 & 0.03692508 & 0.03465282 & 0.03669940 & 0.00128951 \\
\hline 150 & & 1.0 & 0.07599905 & 0.07343580 & 0.07592677 & 0.00125237 \\
\hline 150 & & 2.0 & 0.15142838 & 0.14356337 & 0.15133244 & 0.00134335 \\
\hline
\end{tabular}

Table 4: $\operatorname{RMSE}(\theta)$

\begin{tabular}{|c|c|c|c|c|c|c|}
\hline \multirow{2}{*}{$\mathrm{n}$} & \multirow{2}{*}{$\lambda$} & \multirow{2}{*}{$\theta$} & \multicolumn{4}{|c|}{ Estimation Method } \\
\hline & & & $\mathrm{ML}$ & MOM & Jack & Prop. \\
\hline 15 & 2 & 0.5 & 0.09280074 & 0.09991132 & 0.12707651 & 0.00120577 \\
\hline 15 & & 1.0 & 0.19909425 & 0.21130827 & 0.25449135 & 0.00162497 \\
\hline 15 & & 2.0 & 0.37783125 & 0.40150825 & 0.51122547 & 0.01736784 \\
\hline 15 & 3 & 0.5 & 0.09895411 & 0.09851459 & 0.12521339 & 0.00131140 \\
\hline 15 & & 1.0 & 0.19269626 & 0.20216291 & 0.23466462 & 0.00156591 \\
\hline 15 & & 2.0 & 0.40091586 & 0.39617763 & 0.52286084 & 0.02595584 \\
\hline 15 & 5 & 0.5 & 0.10204577 & 0.10232574 & 0.12227855 & 0.00136207 \\
\hline 15 & & 1.0 & 0.18746573 & 0.20341067 & 0.26705682 & 0.00165824 \\
\hline 15 & & 2.0 & 0.39231337 & 0.41572816 & 0.51116656 & 0.02139605 \\
\hline 30 & 2 & 0.5 & 0.06192158 & 0.06784869 & 0.06183979 & 0.00122210 \\
\hline 30 & & 1.0 & 0.12624426 & 0.13825712 & 0.12612744 & 0.00157423 \\
\hline 30 & & 2.0 & 0.25996604 & 0.29525995 & 0.26735242 & 0.00586760 \\
\hline 30 & 3 & 0.5 & 0.06164153 & 0.07484130 & 0.06088505 & 0.00128101 \\
\hline 30 & & 1.0 & 0.12688509 & 0.14949160 & 0.13175902 & 0.00126936 \\
\hline 30 & & 2.0 & 0.24227728 & 0.28855446 & 0.24867299 & 0.00493893 \\
\hline 30 & 5 & 0.5 & 0.06176586 & 0.07155359 & 0.06223975 & 0.00128989 \\
\hline
\end{tabular}




\begin{tabular}{|l|l|l|l|l|l|l|}
\hline 30 & & 1.0 & 0.12451775 & 0.14306307 & 0.12724643 & 0.00132364 \\
\hline 30 & & 2.0 & 0.24368197 & 0.28847773 & 0.24426256 & 0.00377192 \\
\hline 60 & 2 & 0.5 & 0.04344242 & 0.05237296 & 0.04416112 & 0.00126538 \\
\hline 60 & & 1.0 & 0.08298391 & 0.09552740 & 0.08548191 & 0.00131128 \\
\hline 60 & & 2.0 & 0.16596784 & 0.19105480 & 0.17096348 & 0.00146489 \\
\hline 60 & 3 & 0.5 & 0.04149197 & 0.04776370 & 0.04274120 & 0.00126634 \\
\hline 60 & & 1.0 & 0.08298395 & 0.09552740 & 0.08548643 & 0.00130859 \\
\hline 60 & & 2.0 & 0.16596782 & 0.19105480 & 0.17096200 & 0.00146630 \\
\hline 60 & 5 & 0.5 & 0.04149197 & 0.04776370 & 0.04274109 & 0.00126640 \\
\hline 60 & & 1.0 & 0.08298393 & 0.09552740 & 0.08548315 & 0.00131042 \\
\hline 60 & & 2.0 & 0.16596785 & 0.19105480 & 0.17096194 & 0.00146623 \\
\hline 150 & 2 & 0.5 & 0.02722455 & 0.03436133 & 0.02730857 & 0.00133686 \\
\hline 150 & & 1.0 & 0.05330601 & 0.06470859 & 0.05259551 & 0.00123206 \\
\hline 150 & & 2.0 & 0.10922870 & 0.14078246 & 0.10918481 & 0.00126338 \\
\hline 150 & 3 & 0.5 & 0.02730716 & 0.03519561 & 0.02729417 & 0.00115416 \\
\hline 150 & & 1.0 & 0.04993126 & 0.06380849 & 0.05019086 & 0.00126576 \\
\hline 150 & & 2.0 & 0.10632348 & 0.12906069 & 0.10689809 & 0.00129757 \\
\hline 150 & 5 & 0.5 & 0.02647323 & 0.03440536 & 0.02653836 & 0.00118965 \\
\hline 150 & & 1.0 & 0.05162010 & 0.06520734 & 0.10526452 & 0.00119065 \\
\hline 150 & & 2.0 & 0.10453310 & 0.12786372 & 0.00129332 \\
\hline
\end{tabular}

\section{Future Works:}

i.) The framework of this paper can also be carried out in the context of other probability distributions for the maxima distribution (e.g. lognormal). Another approach can be updated as a recommendation, by more sophisticated proposition in terms of confidence interval $\left\{\varphi_{0 \mathrm{i}} \pm\right.$ uncertainity points $\left.)\right\}$ instead of the interval $\left(\varphi_{0 \mathrm{i}} \pm\right.$ fixed points) to emphasize the scope of the research.

ii.) Nonparametric approaches could be used such as smoothing methods: kernel, splines or wavelet methods to make advanced comparison.

\section{REFERENCES}

Al-Adilee, Ahmed and Mohamed, D. Karrar, 2014. "Linear Regression Model Related to Gumbel Distribution", Journal of Kerbala University, 12(4): 70-78).

Clarke, Robin, T., 2002, "Estimating time trends in Gumbel-distribution data by means of generalized linear models", Water resources research, 38(7): 1-11.

Eforn, B., 1979. "Bootstrap Methods: Another Look at The Jackknife", Annals of Statistics, 7(1): 1-26.

Hall, M.J., H.F.P. Van Den Boogaard, R.C. Fernando and A.C. Mynett, 2004. "The Construction of Confidence Intervals for Frequency Analysis using Resampling Techniques", Hydrology and Earth System Sciences, 8(2): 235-246.

Mahdi, S. and M. Cenac, 2005. "Estimating Parameters of Gumbel distribution using the methods of Moments, Probability weighted moments and Maximum likelihood", Revista de Mathematica: Teoria y Aplicciones, 12(1\&2): (151-156).

Mohammed, Makki A., 2011, "Proposed Method for estimating the scale and location parameters for Gumbel distribution", Magistra, 78: 1-13.

Raynal-Villasenor, Jose A., 2012," Maximum Likelihood Parameter Estimators for The Two Populations GEV Distribution", IJRRAS, 11(3): 350-357.

Yousef, Omar M. and S.A. Al-Subh, 2014. " Estimation of Gumbel Parameters under Ranked Set Sampling", Journal of Modern Applied Statistical Methods, 13(2): 432-443. 